\title{
Computer simulations of charge and steric stabilised colloidal suspensions
}

\author{
Marjolein Dijkstra \\ Debye Institute, Soft Condensed Matter Physics, Utrecht University, Princetonplein 5, 3584 CC Utrecht, The Netherlands
}

\begin{abstract}
Computer simulations of colloidal suspensions are prohibited by slow equilibration as very different length and time scales are involved for the various species. This is the reason that most simulations involve some degree of coarse-graining, whereby the degrees of freedom of the microscopic particles are traced out, and the mesoscopic particles interact with an effective potential, resulting in a coarse-grained, effective one-component description of the suspension. The focus of this paper is on recent simulation work of charge and steric stabilised colloidal suspensions. We discuss both direct simulations of the true colloidal mixture and coarse-grained approaches of the suspension involving effective interactions for the colloids. (C) 2001 Elsevier Science Ltd. All rights reserved.
\end{abstract}

Keywords: Charged colloids; Colloid-polymer mixtures; Hard spheres; Phase separation; Effective potentials; Computer simulation

\section{Introduction}

Colloidal suspensions are complex fluids that consist of mesoscopic particles suspended in a solvent (e.g. water). The colloidal particles are significantly larger than the solvent molecules, but small enough to show Brownian motion. In the case that the linear dimension $R$ of the colloids is in the regime of 10 $\mathrm{nm}<R<1000 \mathrm{~nm}$, no significant sedimentation occurs in the earth's gravity. Examples of colloidal particles are viruses, proteins, synthetic polymeric particles (latex, PMMA), micelles, etc. Suspensions of these particles play an important role in biology, e.g. blood, but also many industrial products are essentially colloidal suspensions, e.g. paints, inks, food, detergents, cosmetics.

Due to fluctuating dipole moments, an attractive 'dispersion' force, or Van der Waals force, acts

E-mail address: m.dijkstra@phys.uu.nl (M. Dijkstra). between every pair of atoms separated by a distance $r$. Summing over all pairs of atoms in two colloidal particles gives rise to a strong Van der Waals attraction between the colloids, which can be larger by orders of magnitude than the thermal energy $k_{B} T$. This may lead to irreversible aggregation of the colloids. In order to stabilise a colloidal suspension against irreversible aggregation two mechanisms are common: charge and steric stabilisation. In the case of charge stabilisation, the colloidal particles have ionisable groups on their surfaces, which dissociate when the particles are suspended in a polar liquid. The colloidal particles then acquire a net surface charge $Z$ e, with $e$ the elementary charge and $Z$ the charge number typically in the range $10^{2}<|Z|<10^{5}$. The released counterions form a diffuse layer of thickness $\lambda_{D}$ around each colloidal particle, where $\lambda_{D}$ is the Debye screening length. The approach of two charged colloids leads to overlap of these so-called electric double layers, and causes a repulsive force that can stabilise the particles against aggregation [1]. 
In the case of steric stabilisation the colloidal particles are coated with a polymer layer. When two coated colloidal particles approach each other sufficiently closely, the polymer layers interpenetrate and overlap, which leads to a reduction of the polymer entropy, and hence to an effective repulsive force between the colloids. This repulsion leads again to stabilisation of the particles against aggregation.

Often other components, such as salt ions, polymers or smaller colloids, are present in suspension as well. In the case of charged colloids, the addition of electrolyte or salt changes the Debye screening length $\lambda_{D}$. On the other hand, the addition of free polymer coils or smaller colloids to a steric stabilised colloidal suspension induces a depletion interaction between colloids which is mainly attractive and of a range of the size of the depletant $[2,3]$. The concentration of added salt, or the size and concentration of added free polymers or smaller colloids can therefore, be used as control parameters with which the effective interactions between the colloids can be tailored. The possibility of tailoring the effective interactions enriches the physics of colloidal systems compared to simple (atomic) fluids, and leads to a wide range of practical applications.

The focus of this review is on computer simulations of steric and charge stabilised colloidal suspensions, with particular emphasis on published research over the past five years. In Section 2, research on charge stabilised colloidal suspensions is discussed. The reader is also referred to an excellent review by Hansen and Löwen on recent theoretical and experimental advances in charged colloids [ $4^{\bullet \bullet}$ ]. Cell model and hypernetted chain calculations of polyelectrolytes are reviewed by Vlachy [5 ${ }^{\bullet \bullet}$ ]. In Section 3 a survey on recent simulation work of steric stabilised suspensions is presented.

\section{Charge-stabilised colloidal suspensions}

Charge-stabilised colloidal suspensions consist of (spherical or anisotropic) mesoscopic colloidal particles suspended in a polar solvent with co- and counterions. The radius of the co- and counterions is comparable to that of the solvent molecules, i.e. of the order of $0.1-0.3 \mathrm{~nm}$. A statistical mechanics description of these highly asymmetric multicomponent fluids represent a major challenge as very different length and time scales are involved for the various species. This is the reason why attempts to treat the mesoscopic colloids and the microscopic salt and solvent particles on an equal footing usually fail. It is therefore not surprising that the present understanding of these systems is based on simplified models, in which the degrees of freedom of the microscopic particles have been integrated out, and the mesoscopic particles interact with an effective (usually pairwise) potential resulting in a coarse-grained effective one-component description of the suspension. The standard and very successful effective one-component description of charged colloidal suspensions dates back to the 1940s and is due to Derjaguin, Landau, Verwey, and Overbeek (DLVO) [2]. The DLVO theory is the corner stone of colloid science; it not only describes the diffuse double layer of thickness $\lambda_{D}$ of co- and counterions surrounding the charge surfaces of colloidal particles, but it also predicts effective screened Coulomb repulsions, with decay length $\lambda_{D}$, between pairs of colloids. On the basis of the DLVO theory one would not expect a gas-liquid transition at salt concentrations of the order of a few $\mu \mathrm{m}$, since it predicts that the Van der Waals attraction - required to provide the cohesive energy of the liquid within the conventional picture - is completely masked by the much longer-ranged electrostatic repulsions. However, in the past few years a variety of unexplained phenomena has been observed in experiments of charge-stabilised colloidal suspensions at low salt concentrations. For instance, vapour-liquid condensation [6], large stable 'voids' in otherwise homogeneous suspensions [7,8] and anomalously long-lived dense clusters [9] are observed in experiments at very low salt concentrations. A long-range attraction between like charged spheres, which would be in contradiction with the DLVO theory, would account naturally for the experimental evidence of gas-liquid coexistence. Indeed the effective pair potential obtained from video microscopy and optical tweezers measurements seem to suggest that the interactions between like-charged colloidal particles in the vicinity of glass walls (not in bulk) are attractive $[9,10]$. However, the nature of these attractions has been disputed and it was shown recently that the attractive interaction can be explained by hydrodynamic effects [11]. The inferred evidence for attractive interactions between likecharged colloidal particles is qualitatively inconsistent with the long-accepted repulsive DLVO potential, generating intense theoretical and experimental activities and vivid debates in this field.

In most theoretical descriptions of colloidal systems, the solvent is regarded as a structureless continuum, characterised by a dielectric constant $\varepsilon$. In this so-called 'Primitive model' description, the charged colloids (macroions) and co- and counterions (microions) are assumed to be charged spheres interacting via short-range potentials and Coulombic interactions. The first simulations of the asymmetric primitive model were performed in the early 1980s [12]. However, even the simplified highly asymmetric 
primitive model gives severe equilibration problems in computer simulations as the number of particles involved in the simulations increases dramatically with larger charge asymmetry and the convergence of the simulations slows down due to the accumulation of microions close to the macroions. Recent progress has been made in simulations of the asymmetric primitive model by Linse et al. who used efficient cluster move algorithms [13 ${ }^{\bullet \bullet}$ ] and the Ewald summation technique $[14,15]$ for handling the long-range interactions. However, the simulations are still limited to charge number $|Z|<160$ describing more the micellar rather than the colloidal regime [16 $\left.{ }^{\bullet \bullet}\right]$. Linse (1999) [17] discusses the efficiency of molecular dynamics and Monte Carlo simulations of the asymmetric primitive model using soft and hard potentials for the macro- and microions.

Using computer simulations of the primitive model, effective attractive forces between like-charged colloids due to electrostatic interactions have been found for divalent and trivalent counterions, but the attractions seem to be of short-range character $\left[16^{\bullet \bullet}\right.$, 18-20]. Short-range attractions due to electrostatic counterion correlations were also found in simulations of charged spheres in divalent counterion solutions [21-23] and in theoretical considerations regarding like-charged plates [24], in Monte-Carlo simulations of lamellar colloids [25,26], in simulations of charged surfaces [27-29], in anisotropic HNC [29] and reference $\mathrm{HNC}$ calculations [30], and in density functional theory calculations using local density and weighted density approximations $\left[31,32^{\bullet \bullet}\right]$. Attractive forces between charged surfaces are even observed in the case of monovalent microions, provided the coions are larger than the counterions [30]. The origin of the short-range attraction is caused by the spatial correlations between the counterions associated with different macroions $\left[16^{\bullet \bullet}, 32\right]$ and was already suggested by Oosawa in the 1960s [34]. Several mechanisms for the effective attractions are proposed in the literature. (1) Correlations keep the counterions apart at the surface of the macroion and limit the piling up. This leads to a reduction of the counterion density at contact, resulting in a lower pressure according to the contact theorem and an effective attraction [ $4^{\bullet \bullet}$ ]. (2) Coulombic interactions between the microions result in an alternation of positive and negative charges at the surface of the macroion. Two macroions approaching each other adjust complementarily to each other resulting in an electrostatic attraction [24]. (3) Charge inversion by an overcompensation of surface charge due to core-core correlations and electrostatic correlations between the microions (several mechanisms are mentioned in [30]). (4) The counterion fluctuations are suppressed close to the macroions, due to counterion accumulation at the macroion sur- face, while these are unconstrained in other regions. This leads to a Casimir-type fluctuation-induced attraction [33].

Simulations of saltfree suspensions of macroions with 60-160 elementary charges and sufficiently strong electrostatic coupling (trivalent counterions), show that the effective short-range attraction can lead to macroscopic phase separation $\left[16^{\bullet \bullet}, 18,19\right]$. No phase separation is observed in simulations with divalent and monovalent counterions. The effect of salt is studied in [19]. Also, Hribar et al. found strong clustering of macroions in a solution of trivalent counterions in his simulations [35]. Attraction between likecharged macroions were also found in Monte Carlo simulations of Allahyarov et al. [36 $6^{\bullet}$. These authors found an attractive effective force for divalent counterions, charge asymmetry of $32: 2$ and size ratio of approximately 22 , and for charge asymmetry of $320: 2$ and size ratio of approximately 198, while good agreement was found with DLVO for moderate charge ratios of approximately $1-4$ and size ratios $\simeq 1\left[37^{\bullet \bullet}\right]$. No attraction was found in the case of monovalent counterions $\left[36^{\bullet}, 38\right]$. The mechanism for this attraction was assigned to Coulombic depletion [36 $6^{\bullet}$. However, Linse et al. found that the short-range attractions are similar for finite-sized counterions and pointlike counterions, and they conclude that the depletion mechanism cannot be responsible for the attraction [20]. Attractive forces were also found in simulations of macroions confined between charged plates at sufficiently strong electrostatic coupling $\left[39,40^{\bullet \bullet}\right]$, while an analytical proof shows that no attractions are possible in the Poisson-Boltzmann approximation [41,42]. It is therefore argued that the attraction between like-charged colloids is due to spatial and temporal fluctuations of counterions as these are neglected in the Poisson-Boltzmann approach $\left[40^{\bullet \bullet}\right]$. The effective triplet forces were calculated between macroions in a triplet-configuration in the form of an equilateral triangle for charge asymmetry of $280: 1$ and size ratio of 1000 [43॰•]. The total net force was always repulsive, but the triplet contribution was attractive. The influence of triplet interactions on the phase behaviour was studied in $\left[44^{\bullet}\right]$.

Finally, we stress again that the molecular nature of the solvent is ignored in the primitive model. To go beyond the description of the primitive model, one can include neutral particles while keeping the dielectric constant $\varepsilon$ in the Coulombic interactions between the charged species. Such a model has been used in Monte Carlo simulations [45] and in Poisson-Boltzmann calculations and hypernetted chain calculations [46]. In [47], the authors claim that an attractive force exists between the macroions as the contact value of the macroion-macroion pair correlation function is 
larger than one at sufficiently large concentrations of the neutral component. A further refinement of the model would include the polar nature of the solvent by using embedded point dipoles or more sophisticated pair potentials for water, like the SPC/E potential [48].

In conclusion, direct simulations of the highly asymmetric primitive model are limited to moderate charge and size asymmetries due to equilibration problems. For the study of equilibrium properties such as phase diagrams, this problem may be overcome by reducing the multi-component suspension consisting of macroions and microions to an effective one-component system containing macroions only. This formal mapping of the multi-component suspension onto an effective one-component system of the colloids can be achieved by integrating out the degrees of freedom of the microions, giving rise to effective interactions for the macroions. In principle, these effective interactions contain one-, two-, threeand higher-body interactions, but is often assumed to be pairwise additive. The essence of this approach is to find reliable effective potentials for the macroions and a big variety of approaches exist. The most sophisticated approach involves a numerical evaluation of equilibrium co- and counterion densities for each macroion configuration during a simulation in the same spirit as the 'ab initio' method of Car and Parrinello for ion-electron systems $\left[49^{\bullet \bullet}, 50,51^{\bullet \bullet}\right]$. In this approach the macroions are treated explicitly in a molecular dynamics simulation, while the microions are treated in a coarse-grained description using the framework of density functional theory. Here the equilibrium microion density profiles are obtained by minimising the grand potential functional. The grand potential functional consists of an ideal part, an external field, and an excess part. The ideal part and external field contributions are known exactly, and the excess part is assumed to consist of a Coulombic contribution and a correlation part. The effective forces between the colloids are calculated 'on the fly' for each colloid configuration via the classical version of the Hellmann-Feynman Theorem [49 $\bullet$ ]. It is important to note that, in principle, the effective forces depend on all co-ordinates of the colloids, i.e. involve pair-, triplet-, and higher-body terms. This procedure has been applied to saltfree suspensions of charged spherical colloids $\left[49^{\bullet \bullet}, 50,51^{\bullet \bullet}, 52,53\right]$, to colloids with added salt [54], to charged colloidal rods [55,56], to clay suspensions [57] and to polyelectrolytes [58]. Alternatively, one can use a simpler or approximate grand potential functional and derive the effective interactions between the colloids in advance and use these interactions in a simulation or in theory. Such a procedure has been applied in $\left[59,60^{\bullet \bullet}, 61,62\right]$. In this work, the grand potential functional is simplified by ignoring the correlations and expanding the local densities in the ideal term to second order about the bulk densities. The grand potential functional can now be minimised analytically and leads back to solving the linearized Poisson-Boltzmann equation. The effective interaction for the colloids contains only pair interactions, which is precisely the well known DLVO potential [1], and more surprisingly it consists of a statedependent volume term $\left[59,60^{\bullet \bullet}, 61,62\right]$. This term has been generally overlooked, but a simple variational calculation of the free energy of this effective onecomponent system yields surprisingly rich predictions of gas-liquid, gas-solid, and liquid-solid coexistence, with triple and critical points, at extremely low salt concentrations $\left[59,60^{\bullet \bullet}, 61,62\right]$. At higher salt concentrations, where DLVO is known to be fine, it yields results equivalent to the DLVO theory. The phase instability at low salt concentrations is thus driven by the volume term, while the macroion pair interaction is repulsive, consistent with DLVO. An alternative interpretation for the volume term is in terms of attractive many-body (triplet or higher-body) interac-

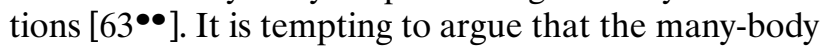
interactions are lumped together in the volume terms leading to a non-trivial density-dependent effective interaction, which warrants a more detailed investigation $\left[64^{\bullet}\right]$.

The phase diagram of particles interacting via only the DLVO pair potential is determined by simulations $\left[65^{\bullet \bullet}, 66-68\right]$. For completeness, we also mention the simulations of Tata et al. They explain the observed experimental phase behaviour of charged colloids (void formation, liquid-gas transition, etc.) by simulations using the Sogami-Ise potential $[8,69,70]$. This effective pair interaction [71] contains a longrange attractive component, but lacks rigorous theoretical motivation.

In summary, the unexplained experimental observations (vapour-liquid coexistence, void structures, etc.) in charged colloids have generated many theoretical and experimental activities in this field. Over the past few years the published research on charged colloids has expanded enormously and it is therefore an impossible task to review them all properly. It was intended to give the reader a flavour of the rapidly growing literature on charged colloids and stress again that the focus of this review was on computer simulations. Despite the amount of work spent on charged colloids, it is still not clear what the origin is of the experimental observations, like void formation and gas-liquid or gas-solid coexistence. Future work should focus on understanding the origin of the attractive component in the effective pair interaction due to correlation effects of the microions, which are observed in simulations of the asymmetric primitive model. At the moment, several mechanisms are pro- 
posed for the effective attractions between macroions due to microion-microion correlations. These microion correlations are not captured in the (linearized) Poisson-Boltzmann mean field theories and thus also not included in the approaches that involve a coarse-grained description for the microion using a density functional theory that reduces to (linearized) Poisson-Boltzmann. It is interesting to investigate how to incorporate the correlations effects in a density functional and to see what the influence is on the colloid stability. One should also investigate if there is a connection between the many-body interactions and the so-called volume terms. Finally, studies of the interfacial behaviour of charged colloids might give us some insight in the formation of voids and biphasic regions.

\section{Steric-stabilised colloidal suspensions}

In the case of steric stabilisation, the colloidal particles are coated with a polymer layer, which leads to a steep repulsive interaction between the colloids when they approach each other; the colloidal pair potential can therefore be regarded as hard-sphere like. The hard-sphere system has been studied in great detail. In 1957 Wood and Jacobson [72] and Alder and Wainwright [73] showed by computer simulations that a system of purely repulsive hard spheres has a well-defined freezing transition. These results were disputed for a long period, but nowadays it is generally accepted that a system of identical hard spheres does have a fluid-solid transition [74]. The origin of this freezing transition is purely entropic and occurs because the entropy of the crystalline phase is higher than that of the fluid phase at sufficiently high densities. The location of the hard-sphere freezing transition was determined from simulations by Hoover and Ree, who found that the packing fractions of the coexisting fluid and face-centred cubic (fcc) solid phase are given by $\eta_{\text {fluid }}=0.494$ and $\eta_{\text {solid }}=0.545$, which corresponds to a pressure $P \sigma^{3} / k_{B} T=11.69$ with $\sigma$ the diameter of the hard spheres [75]. Finite size effects were studied by Wilding et al. using a novel Monte Carlo phase switch method [76 ${ }^{\bullet}$. It has also been shown recently that at co-existence, the fcc crystal is indeed more stable than the hexagonal close-packed (hcp) crystal [77-79]. Often other components, such as smaller steric stabilised colloids or non-adsorbing polymer coils are present in suspension as well. An overview of work spent on the addition of smaller steric stabilised colloids is presented in Section 3.1 and colloid-polymer mixtures are discussed in Section 3.2.

\subsection{Colloid-colloid mixtures}

In the case of mixtures of large and small colloidal particles, the system can be regarded as a binary hard-sphere mixture. In the last decade, it was found that binary hard-sphere mixtures show extremely rich phase behaviour. Spindle, azeotropic, and eutectic type of phase diagrams and complex crystalline superlattice structures are found in theory, simulation, and experiments, when the size ratio $q \equiv \sigma_{2} / \sigma_{1}>0.4$. The diameters of the large and small spheres are, respectively, $\sigma_{1}$ and $\sigma_{2}$. Published work in this size ratio regime is reviewed in $\left[80^{\bullet \bullet}\right]$. When the size ratio is more asymmetric, the addition of the second component leads to the so-called depletion effect. This effect induces effective attractions between large colloids at small separations due to an unbalanced pressure of small (polymeric) spheres. An alternative description of the same effect is that free volume of the small spheres is gained due to the overlap of excluded volume of clustering large spheres; the resulting gain of entropy of the small spheres drives this clustering, and thus induces effective attractions between the large spheres.

The main issue here is whether the depletion effect, which is known to lead to demixing in colloidpolymer mixtures [2,3], is sufficiently strong to drive demixing in a binary fluid mixture of large and small hard spheres or whether the additive mixture of hard spheres is miscible for all size ratios and compositions. The discussion was instigated in 1991 by Biben and Hansen, who showed within integral equation theory that the binary hard-sphere mixture exhibits a spinodal instability in the high-density fluid when the size ratio of the two species is more extreme than 1:5 [81]. As this result was in contradiction with a classic study by Lebowitz and Rowlinson, who had concluded that the mixture is stable against demixing regardless of the state point and the size ratio [82], it initiated renewed interest in this system.

One might suppose that computer simulations should have resolved the issues concerning the phase behaviour. However, direct simulations of highly asymmetric binary mixtures are prohibited by slow equilibration when the packing fraction of the small spheres becomes substantial and there have been no systematic attempts to calculate phase diagrams for the asymmetric cases which are of most interest. Some progress has been made by cluster move techniques based on the configurational bias Monte Carlo method $\left[83,84^{\bullet}\right]$ and by a recently developed algorithm [85'], but the statepoints of interest (high small sphere packing fractions) could still not be reached. A (meta)stable demixing transition in the fluid phase 
does occur in simulations and theories of binary mixtures of hard parallel cubes [86], binary mixtures of freely rotating hard rods [87], non-additive hardsphere mixtures [88], and polydisperse mixtures of hard spheres [89].

On the theoretical side it is now well-accepted that those approaches which attempt to treat both species on an equal footing, e.g. integral equation theories or virial expansions of the mixture equation of state, yield results which are notoriously sensitive to the details of the approximations. The (non-)existence and location of the spinodal instability is particularly susceptible [90-92], but also the location of the fluid-solid phase boundary is very sensitive to the choice of approximation [93-99]. Experiments show that the demixing transition is strongly coupled to the freezing transition $\left[100-104^{\bullet \bullet}\right]$.

Therefore, a different strategy is often followed, in which advantage is taken of the large size asymmetry. In this approach the binary mixture is formally mapped onto an effective one-component system by integrating out the degrees of freedom of the small species in the partition function $\left[80^{\bullet \bullet}, 105^{\bullet \bullet}, 106^{\bullet \bullet}\right]$. An effective Hamiltonian is then obtained for the larger ones, which consists of zero-body, one-body, two-body and higher-body interactions. The effective two-body interactions or depletion potentials have been calculated within hypernetted-chain-based approximations [108], in simulations $\left[109^{\bullet \bullet}, 110^{\bullet \bullet}, 111^{\bullet \bullet}, 112\right]$, using a virial expansion $\left[113^{\bullet}\right]$, integral equation theory $[114,115]$, and density functional theory $\left[110^{\bullet \bullet}\right.$, $\left.116,117^{\bullet \bullet}, 118,119^{\bullet \bullet}\right]$. Depletion potentials have also been 'measured' experimentally [120].

Using this approach and ignoring three-body and higher-body interactions, simulations show the existence of a fluid-fluid demixing transition for size ratios of $1: 10$ or more extreme $\left[80^{\bullet \bullet}, 105^{\bullet \bullet}, 106^{\bullet \bullet}\right]$. However, this fluid-fluid transition is metastable with respect to the fluid-solid transition that turns out to occur at strikingly low values of the packing fraction of the large spheres. More surprisingly, also an isostructural solid-solid transition at high packing fractions of the large spheres is found. This transition becomes stable for a size ratio $<0.05$. Good agreement is found with direct simulations of the true binary hard-sphere mixture $\left[80^{\bullet \bullet}, 105^{\bullet \bullet}\right]$, thereby justifying the depletion potential picture. Similar results are found in other simulations [121 ${ }^{\bullet \bullet}$ ] and theories $[122,123]$ of the effective one-component system, while gelation of binary hard-sphere mixtures is studied in [124] and structure in [125]. The structure of the true binary hard-sphere mixtures has been studied extensively for moderate size ratios by integral equation theories and by simulations, and for these the reader is referred to $\left[80^{\bullet \bullet}\right]$ and references therein.

\subsection{Colloid-polymer mixtures}

Experiments on colloid-polymer mixtures show that the phase behaviour depends on the size ratio of colloids and polymer (see [126 ${ }^{\bullet}$ ] and references, therein). For sufficiently large polymer coils (compared to the colloids) a stable fluid-fluid and a three-phase coexistence of a colloidal gas, liquid, and solid were found. Structure of colloid-polymer mixtures is studied by integral equation theories using the polymer reference interaction site model $\left[127,128^{\bullet}, 129\right]$. Direct simulations of colloid-polymer mixtures are prohibited as long relaxation times are required for mixtures and for polymeric systems. One way to alleviate this problem is to represent the polymers by ideal lattice chains and the colloids by hard spheres $\left[129^{\bullet \bullet}\right]$. Simulations of this system show that indeed the phase behaviour depends critically on the size ratio of the polymer and colloid, consistent with experiments $\left[130^{\bullet \bullet}\right]$. Another way to circumvent the problem of slow equilibration is to ignore the degrees of freedom of the polymer chains. A simple idealised model, which is often used for a suspension of sterically stabilised colloidal particles and nonadsorbing polymers, is the so-called Vrij-AsakuraOosawa model. This is an extremely non-additive binary hard-sphere model in which the colloids are treated as hard spheres with diameter $\sigma_{1}$ and the interpenetrable, non-interacting polymer coils in a theta-solvent are treated as point particles but which are excluded from the colloids to a centre-of-mass distance of $\left(\sigma_{1}+\sigma_{2}\right) / 2$. The diameter of the polymer coil is $\sigma_{2}=2 R_{g}$ with $R_{g}$ the radius of gyration of the polymer. Direct simulation of the VrijAsakura-Oosawa model is again prohibited by slow equilibration, as huge number of polymers are needed per colloid particle at the state-points of interest. Direct simulations of the Vrij-Asakura-Oosawa model are only performed for systems in which the polymers are represented by ideal particles on a lattice $\left[130^{\bullet \bullet}\right]$. The structure of the Vrij-Asakura-Oosawa model is determined within integral equation theory $\left[64^{\bullet}, 131^{\bullet}\right]$ and the phase diagram is studied using the freevolume approach $\left[133^{\bullet}\right]$. Recently, a density functional theory was designed specifically for the binary Vrij-Asakura-Oosawa model. The structure, adsorption at a hard wall, and the fluid-fluid interface are studied within this density functional theory and are compared with simulations $\left[134^{\bullet \bullet}\right]$.

A different strategy is to derive an effective onecomponent Hamiltonian for the colloids by formally integrating out the degrees of freedom of the polymer $\left[135^{\bullet \bullet}\right]$. The effective Hamiltonian consists of zerobody, one-body, two-body, and higher-body terms. The two-body term or depletion potential is determined from simulations $\left[136^{\bullet \bullet}, 137^{\bullet}, 138^{\bullet}\right]$ and field theory 
[139॰]. The depletion potentials for non-additive hard-sphere mixture are determined from density functional theory $\left[140^{\bullet}\right]$. The depletion potential has been 'measured' recently in experiments [141-144]. In the case of the Vrij-Asakura-Oosawa model, the two-body term is precisely that given by the VrijAsakura-Oosawa pair potential [2,3]. Moreover, for size ratios $q \equiv \sigma_{2} / \sigma_{1} \leq 0.154$, three-body and higherbody terms are identically zero, so the mapping to the two-body approximation to this effective Hamiltonian is exact in this regime. Monte Carlo simulations for the Vrij-Asakura-Oosawa model are performed using the effective Hamiltonian truncated at the pairwise term. The phase behaviour and structure are determined of the effective one-component system for size ratios $q=0.1,0.4,0.6$ and 0.8 , respectively [135 ${ }^{\bullet \bullet}$ ], and are compared with results from perturbation theory of the effective Hamiltonian $\left[135^{\bullet \bullet}, 145^{\bullet}\right]$ and with the phase diagram from the free-volume approach $\left[133^{\circ}\right]$. Adsorption at a hard wall is studied in simulations and compared with density functional theory [146 ${ }^{\bullet \bullet}$ ]. The interfacial behaviour of colloid-polymer mixtures is studied using the effective Hamiltonian in density functional theory [147] and the nonequilibrium behaviour and kinetics is studied using Brownian dynamics simulations [148 ${ }^{\bullet}$. The phase diagram and structure of the Vrij-Asakura-Oosawa model is recently determined from simulations using the full effective Hamiltonian for $q=1.0$ [149 ${ }^{\bullet \bullet}$ ], but these results could not explain the colloidal structure factors found in recent light scattering experiments [150].

In summary, direct simulations of colloid-polymer mixtures are prohibited due to slow equilibration and one is often forced to make drastic approximations. Often the Vrij-Asakura-Oosawa model is used as a simple model for suspensions of sterically stabilised colloidal particles and non-adsorbing polymers. The phase behaviour and structure of the VrijAsakura-Oosawa model obtained from theory and simulation agree well with experiments, but show large deviations in the regime of similar size of colloids and polymers. Future work should focus on models using a more realistic description of the polymer and its interaction with the colloids. It is interesting to derive depletion potentials for such a model and to investigate the influence on the phase behaviour and structure.

\section{Conclusions}

Direct computer simulations of colloidal suspensions are limited to moderate size and charge asymmetries due to equilibration problems as very different length and time scales are involved for the various species. One is often forced to resort to simulations that involve some degree of coarse-graining, whereby the degrees of freedom of the microscopic particles (microions, non-adsorbing polymer, smaller colloids, or solvent molecules) are integrated out, and the colloidal particles interact with effective potentials. In this paper, recent simulations are discussed on charge and steric stabilised colloidal suspensions. We focus both on direct simulations of the true colloidal mixture and on coarse-grained approaches of the suspension involving effective interactions for the colloids. These effective interactions can be obtained from (density functional) theory and direct simulations of the true colloidal mixture.

\section{Acknowledgements}

I wish to thank R. van Roij for a critical reading of this manuscript and for stimulating discussions.

\section{References and recommended reading}

- of special interest

$\bullet$ of outstanding interest

[1] Verwey EJW, Overbeek JThG. Theory of the stability of lyotropic colloids. Amsterdam: Elsevier, 1948. Republication Dover Publications, New York (1999)

[2] Asakura S, Oosawa F. On interaction between two bodies immersed in a solution of macromolecules. J Chem Phys 1954;22:1255-1256.

[3] Vrij A. Polymers at interfaces and the interactions in colloidal dispersions. Pure Appl Chem 1976;48:471-483.

[4] Hansen JP, Löwen H. Effective interactions between electric

• double layers. Annu Rev Phys Chem 2000;51:209-242.

[5] Vlachy V. Ionic effects beyond Poisson-Boltzmann theory.

- Annu Rev Phys Chem 1999;50:145-165.

[6] (a) Tata BVR, Rajalakshmi M, Arora. AK. Vapor-liquid condensation in charged colloidal suspensions. Phys Rev Lett 1992;69:3778-3781 See also Erratum. Phys Rev Lett 70:2823 (1993); (b) Palberg T, Würth M. Comment on 'Vapor-liquid condensation in charged colloidal suspensions'. Phys Rev Lett 1994;72:786 See also Erratum. Phys Rev Lett 70:2823 (1993). Tata and Arora reply, Phys Rev Lett $72: 787$ (1994).

[7] Ito K, Yoshida H, Ise N. Void structure in colloidal dispersions. Science 1994;263:66-68.

[8] Tata BVR, Yamahara E, Rajamani PV, Ise N. Amorphous clustering in highly charged dilute poly(chlorostyrenestyrene sulfonate) colloids. Phys Rev Lett 1997;78: 2660-2663.

[9] (a) Larsen AE, Grier DG. Like-charge attractions in metastable colloidal crystallites. Nature 1997;385:230-233; (b) Grier DG. Colloids. a surprisingly attractive couple. Nature 1998;393:621-623.

[10] Kepler GM, Fraden S. Attractive potential between confined colloids at low ionic strength. Phys Rev Lett 1994;73:356-359.

[11] Squires TM, Brenner. MP. Like-charge attraction and hydrodynamic interaction. Phys Rev Lett 2000;85:4976-4979.

[12] Linse P, Jönsson. B. A Monte Carlo study of the electrostatic interaction between highly charged aggregates. A 
test of the cell model applied tomicellar systems. J Chem Phys 1983;78:3167-3176.

[13] Lobaskin V, Linse P. Simulation of an asymmetric electrolyte

- $\quad$ with charge asymmetry 60:1 using hard-sphere and softsphere models. J Chem Phys 1999;111:4300-4309.

[14] (a) de Leeuw SW, Perram JW, Smith. ER. Simulation of electrostatic systems in periodic boundary conditions. I. Lattice sums and dielectric constants. Proc R Soc London Ser A 1980;373:27-56; (b) de Leeuw SW, Perram JW, Smith. ER. Simulation of electrostatic systems in periodic boundary conditions. II. Equivalence of boundary conditions. Proc R Soc London Ser A 1980;373:56-66; (c) de Leeuw SW, Perram JW, Smith. ER. Simulation of electrostatic systems in periodic boundary conditions. III. Further theory and applications. Proc R Soc London Ser A 1983;388:177-193.

[15] Lobaskin V, Linse. P. Accurate simulation of highly asymmetric electrolytes with charge asymmetry 20:1 and 20:2. J Chem Phys 1998;109:3530-3541.

[16] Linse P. Structure, phase stability, and thermodynamics in

-• charged colloidal solutions. J Chem Phys 2000;113: 4359-4373.

[17] Linse P. On the convergence of simulation of asymmetric electrolytes with charge asymmetry 60:1. J Chem Phys 1999;110:3493-3501.

[18] Linse P. Electrostatic attraction and phase separation in solutions of like-charged colloidal particles. Phys Rev Lett 1999;83:4208-4211.

[19] Linse P. Electrostatic attraction and phase separation in solutions of like-charged colloidal particles. J Chem Phys 2000;112:3917-3927.

[20] Lobaskin V, Lyubartsev A, Linse P. Effective macroionmacroion potentials in asymmetric electrolytes. Phys Rev E 2001;63:020401-1 R-020401-4 R.

[21] Grønbech-Jensen N, Beardmore KM, Pincus P. Interactions between charged spheres in divalent counterion solution. Physica A 1998;261:74-81.

[22] Hribar B, Vlachy V. Evidence of electrostatic attraction between equally charged macroions induced by divalent counterions. J Phys Chem B 1997;101:3457-3459.

[23] Wu JZ, Bratko D, Blanch HW, Prausnitz JM. Monte Carlo simulation for the potential of mean force between ionic colloids in solutions of asymmetric salts. J Chem Phys 1999; 111:7084-7094.

[24] Rouzina I, Bloomfield VA. Macroion attraction due to electrostatic correlation between screening counterions. 1. Mobile Surface-adsorbed ions and diffuse ion cloud. J Phys Chem 1996;100:9977-9989.

[25] Pellenq. RJM, Caillol JM, Delville A. Electrostatic attraction between two charged surfaces: A $(\mathrm{N}, \mathrm{V}, \mathrm{T})$ Monte Carlo simulation. J Phys Chem B 1997;101:8584-8594.

[26] Delville A, Levitz. P. Direct derivation of the free energy of two charged lamellar colloids from $(\mathrm{N}, \mathrm{V}, \mathrm{T})$ Monte Carlo simulations. J Phys Chem B 2001;105:663-667.

[27] Guldbrand L, Jönsson B, Wennerström H, Linse. P. Electrical double layer forces. A Monte Carlo study. J Chem Phys 1984;80:2221-2228.

[28] Valleau JP, Ivkov R, Torrie GM. Colloid stability: the forces between charged surfaces in an electrolyte. J Chem Phys 1991;95:520-532.

[29] Kjellander R, Åkesson T, Jönsson B, Marcelja S. Double layer interactions in mono- and divalent electrolytes: a comparison of the anisotropic hypernetted chain theory and Monte Carlo simulations. J Chem Phys 1992;97:1424-1431.

[30] Greberg H, Kjellander R. Charge inversion in electric double layers and effects of different sizes for counterions and coions. J Chem Phys 1998;108:2940-2953.

[31] Stevens MJ, Robbins MO. Density functional theory of ionic screening - when do like charges attract. Europhys Lett 1990;12:81-86.

[32] Tang Z, Scriven LE, Davis HT. Interactions between primi-

- tive electrical double layers. J Chem Phys 1992;97: 9258-9266.

[33] Kardar M, Golestanian R. The friction of vacuum, and other fluctuation-induced forces. Rev Mod Phys 1999;71: $1233-1245$.

[34] Oosawa F. Biopolymers 1968;6:1633.

[35] Hribar B, Vlachy V. Clustering of macroions in solutions of highly asymmetric electrolytes. Biophys J 2000;78:694-698.

[36] Allahyarov E, D'Amico I, Löwen H. Attraction between

- like-charged macroions by Coulomb depletion. Phys Rev Lett 1998;81:1334-1337.

[37] Allahyarov E, Löwen H, Trigger S. Effective forces between

- macroions: the cases of asymmetric macroions and added salt. Phys Rev E 1998;57:5818-5824.

[38] D'Amico I, Löwen H. Effective forces between macroions: a Monte Carlo study. Physica A 1997;237:25-30.

[39] Allahyarov E, D'Amico I, Löwen. H. Effect of geometrical confinement on the interaction between charged colloidal suspensions. Phys Rev E 1999;60:3199-3210.

[40] Terao T, Nakayama T. Effective interaction between highly

- $\quad$ charged colloidal particles under geometrical confinement. J Phys: Condens Matt 2000;12:5169-5177.

[41] Neu JC. Wall-mediated forces between like-charged bodies in an electrolyte. Phys Rev Lett 1999;82:1072-1074.

[42] Sader JE, Chan DYC. Long-range electrostatic attractions between identically charged particles in confined geometries: an unresolved problem. J Colloid Interface Sci 1999; 213:268-269.

[43] Löwen H, Allahyarov. E. The role of effective triplet interac-

- $\quad$ tions in charged colloidal suspensions. J Phys Condens Mater 1998;10:4147-4160.

[44] Wu JZ, Bratko D, Blanch HW, Prausnitz JM. Effect of

- three-body forces on the phase behavior of charged colloids. J Chem Phys 2000;113:3360-3365.

[45] Reščič J, Vlachy V, Bhuiyan LB, Outhwaite CW. Monte Carlo simulations of a mixture of an asymmetric electrolyte and a neutral species. Mol Phys 1998;95:233-242.

[46] Bhuiyan LB, Outhwaite CW. Polyelectrolyte solutions using a three component mixture. Mol Phys 1996;87:625-635.

[47] Vlachy V, Bhuiyan LB, Outhwaite CW. Asymmetric electrolyte in mixture with a neutral component: effects of counterion charge. Mol Phys 1997;90:553-561.

[48] Berendsen HJC, Grigera JR, Straatsma. TP. J Phys Chem 1987;91:6269.

[49] Löwen H, Hansen JP, Madden. PA. Non-linear counterion

-• screening in colloidal suspensions. J Chem Phys 1993; 98:3275-3289.

[50] Löwen H, Hansen JP, Madden. PA. Ab initio description of counterion screening in colloidal suspensions. Phys Rev Lett 1992;68:1081-1084.

[51] Fushiki M. Molecular-dynamics simulation for charged col-

•- loidal dispersions. J Chem Phys 1992;97:6700-6713.

[52] Tehver R, Ancilotto F, Toigo F, Koplik J, Banavar. JR. Absence of many-body effects in interactions between charged colloidal particles. Phys Rev E 1999;59: R1335-R1338.

[53] Löwen H, D'Amico I. Testing of pseudopotentials used in classical Car-Parrinello simulations. J Phys Condens Matt 1997;9:8907-8919.

[54] Löwen H, Kramposthuber G. Optimal effective pair potential for charged colloids. Europhys Lett 1993;23:673-678.

[55] Löwen $H$. Interaction between charged rodlike colloidal particles. Phys Rev Lett 1994;72:424-427. 
[56] Löwen H. Charged rodlike colloidal suspensions - Ab initio approach. J Chem Phys 1994;100:6738-6749.

[57] M. Dijkstra: unpublished.

[58] Hansen JP, Smargiassi E. In: Binder K, Ciccotti G, editors. Monte Carlo and molecular dynamics of condensed matter systems. Bologna: SIF, 1995.

[59] van Roij R, Hansen JP. Van der Waals-like instability in suspensions of mutually repelling charged colloids. Phys Rev Lett 1997;79:3082-3085.

[60] van Roij R, Dijkstra M, Hansen JP. Phase diagram of

- charge-stabilized colloidal suspensions. van der Waals instability without attractive forces. Phys Rev E 1999; 59:2010-2025.

[61] van Roij R, Evans. R. Phase equilibria in a model of low-salt suspensions of charged colloids. J Phys Condens Matt 1999;11:10047-10060.

[62] van Roij R. Attraction or repulsion between charged colloids? A connection with Debye-Hückel theory. J Phys Condens Matt 2000;12:A263-A267.

[63] Warren PB. A theory of void formation in charge-stabilized

-• colloidal suspensions at low ionic strength. J Chem Phys 2000;112:4683-4698.

[64] Dijkstra M, van Roij R, Evans R. Effective interactions,

- $\quad$ structure, and isothermal compressibility of colloidal suspensions. J Chem Phys 2000;113:4799-4807.

[65] Robbins MO, Kremer K, Grest GS. Phase diagram and

-• dynamics of Yukawa systems. J Chem Phys 1988;88: 3286-3312.

[66] Hamaguchi S, Farouki RT, Dubin DHE. Phase diagram of Yukawa systems near the one-component-plasma limit revisited. J Chem Phys 1996;105:7641-7647.

[67] Meijer EJ, El Azhar F. Novel procedure to determine coexistence lines by computer simulation. Application to hardcore Yukawa model for charge-stabilized colloids. J Chem Phys 1997;106:4678-4683.

[68] El Azhar F, Baus M, Ryckaert JP, Meijer. EJ. Line of triple points for the hard-core Yukawa model: a computer simulation study. J Chem Phys 2000;112:5121-5126.

[69] Tata BVR, Arora AK, Valsakumar MC. Reentrant phase transitions in charged colloidsal suspensions: a Monte Carlo study. Phys Rev E 1993;47:3404-3411.

[70] Tata BVR, Ise N. Homogeneous to inhomogeneous transition in charged colloids. Phys Rev B 1996;54:6050-6053.

[71] Sogami I, Ise N. On the electrostatic interaction in macroionic solutions. J Chem Phys 1984;81:6320-6332.

[72] Wood WW, Jacobson JD. Preliminary results from a recalculation of the Monte Carlo equation of state of hard spheres. J Chem Phys 1957;27:1207-1208.

[73] Alder BJ, Wainwright TE. Phase transitions for a hard sphere system. J Chem Phys 1957;27:1208-1209.

[74] Percus JK. The many-body problem. New York: Interscience, 1963.

[75] Hoover WG, Ree. FH. Melting transition and communal entropy for hard spheres. J Chem Phys 1968;49:3609-3617.

[76] Wilding NB, Bruce AD. Freezing by Monte Carlo phase - $\quad$ switch. Phys Rev Lett 2000;85:5138-5141.

[77] (a) Woodcock LV. Entropy difference between the facecentred cubic and hexagonal close-packed crystal structures. Nature 1997;385:141-143; (b) Bolhuis PG, Frenkel D, Mau S-C, Huse DA. Entropy difference between crystal phases. Nature 1997;388:235-236; (c) Woodcock LV. Entropy difference between crystal phases - reply. Nature 1997; 388:236-237.

[78] Bruce AD, Wilding NB, Ackland GJ. Free energy of crystalline solids: a lattice-switch Monte Carlo method. Phys Rev Lett 1997;79:3002-3005.
[79] Bruce AD, Jackson AN, Ackland GJ, Wilding. NB. Latticeswitch Monte Carlo method. Phys Rev E 2000;61:906-919.

[80] Dijkstra M, van Roij R, Evans R. Phase diagram of highly

-• asymmetric binary hard-sphere mixtures. Phys Rev E 1999;59:5744-5771.

[81] (a) Biben T, Hansen JP. On the structure of hard-sphere suspensions in a discrete solvent. Europhys Lett 1990;12:347-352; (b) Biben T, Hansen JP. Phase separation of asymmetric binary hard-sphere fluids. Phys Rev Lett 1991;66:2215-2218; (c) Biben T, Hansen JP. Spinodal instability of suspensions of large spheres in a fluid of small spheres. J Phys: Condens Matter 1991;3:F65-F72.

[82] Lebowitz JL, Rowlinson JS. Thermodynamic properties of mixtures of hard spheres. J Chem Phys 1964;41:133-138.

[83] Biben T. Ph.D. thesis. Université Claude Bernard-Lyon 1, France, 1993.

[84] Dijkstra M, van Roij R. Entropy-drive demixing in binary

- $\quad$ hard-core mixtures: from hard spherocylinders towards hard spheres. Phys Rev E 1997;56:5594-5602.

[85] Buhot A, Krauth W. Numerical solution of hard-core mixtures. Phys Rev Lett 1998;80:3787-3790.

[86] (a) Dijkstra M, Frenkel D, Hansen JP. Phase separation in binary hard-core mixtures. J Chem Phys 1994;101:3179-3189; (b) Dijkstra M, Frenkel D. Evidence for entropy-driven demixing in hard-core fluids. Phys Rev Lett 1994;72:298-300; (c) Martinez-Raton Y, Cuesta JA. Fundamental measure theory for mixtures of parallel hard cubes. II. Phase behavior of the one-component fluid and of the binary mixture. J Chem Phys 1999;111:317-327; (d) Martinez-Raton Y, Cuesta JA. Phase behavior of additive binary mixtures in the limit of infinite asymmetry. Phys Rev E 1998;58:R4080-R4083; (e) Cuesta JA, Martinez-Raton Y. Fundamental measure theory for mixtures of parallel hard cubes. 1. General formalism. J Chem Phys 1997;107:6379-6389; (f) Cuesta JA, Martinez-Raton Y. Dimensional crossover of the fundamental-measure functional for parallel hard cubes. Phys Rev Lett 1997;78:3681-3684; (g) Cuesta JA. Fluid mixtures of parallel hard cubes. Phys Rev Lett 1996;76:3742-3745.

[87] van Roij R, Mulder B, Dijkstra M. Phase behavior of binary mixtures of thick and thin hard rods. Physica A 1998;261:374-390.

[88] (a) Dijkstra M. Phase behavior of non-additive hard-sphere mixtures. Phys Rev E 1998;58:7523-7528; (b) Louis AA, Finken R, Hansen JP. Crystallization and phase separation in non-additive binary hard-sphere mixtures. Phys Rev $\mathrm{E}$ 2000;61:R1028-R1031.

[89] (a) Cuesta JA. Demixing in a single-peak distributed polydisperse mixture of hard spheres. Europhys Lett 1999; 46:197-203; (b) Warren PB. Fluid-fluid phase separation in hard spheres with a bimodal size distribution'. Europhys Lett 1999;46:295-300.

[90] Lekkerkerker HNW, Stroobants A. On the spinodal instability of highly asymmetric hard sphere suspensions. Physica A 1993;195:387-397.

[91] Amokrane S, Regnaut C. Free-volume fraction in hardsphere mixtures and the osmotic spinodal curve. Phys Rev E 1996;53:1990-1993.

[92] (a) Rosenfeld Y. Phase-separation of asymmetric binary hard-sphere fluids - self-consistent density-functional theory. J Phys Chem 1995;99:2857-2864; (b) Rosenfeld Y. Phase-separation of asymmetric binary hard-sphere fluids - self-consistent density-functional theory. Phys Rev Lett 1994;72:3831-3834.

[93] Poon WCK, Warren. PB. Phase behaviour of hard-sphere mixtures. Europhys Lett 1994;28:513-518. 
[94] Dinsmore AD, Warren PB, Poon WCK, Yodh AG. Fluid-solid transitions on walls in binary hard-sphere mixtures. Europhys Lett 1997;40:337-342.

[95] $\mathrm{Xu} \mathrm{H}$, Barentin C. Freezing of very asymmetric binary hard-sphere mixtures. J Phys: Condens Matter 1995; 7:L13-L17.

[96] Caccamo C, Pellicane G. RY theory investigation of phase coexistence in hard sphere mixtures. Physica A 1997; 235:149-158.

[97] Saija F, Giaquinta PV. Statistical entropy of a binary hardsphere mixture: the low-density limit. J Phys: Condens Matter 1996;8:8137-8144.

[98] (a) Coussaert T, Baus M. Virial Approach to hard-sphere demixing. Phys Rev Lett 1997;79:1881-1884; (b) Coussaert T, Baus M. Erratum on 'Virial approach to hard-sphere demixing. Phys Rev Lett 1998;80:4832-4833; (c) Coussaert T, Baus M. Demixing vs. freezing of binary hard-sphere mixtures. J Chem Phys 1998;109:6012-6020.

[99] Vega C. Structure and phase diagram of mixtures of hard spheres in the limit of infinite size ratio. J Chem Phys 1998;108:3074-3075.

[100] Sanyal S, Easwar N, Ramaswamy S, Sood AK. Phase separation in binary nearly-hard-sphere colloids: evidence for the depletion force. Europhys Lett 1992;18:107-110.

[101] van Duijneveldt JS, Heinen AW, Lekkerkerker HNW. Phase-separation in bimodal dispersions of sterically stabilized silica particles. Europhys Lett 1993;21:369-374.

[102] Kaplan PD, Rouke JL, Yodh AG, Pine DJ. Entropically driven surface-phase separation in binary colloidal mixtures. Phys Rev Lett 1994;72:582-585.

[103] Dinsmore AD, Yodh AG, Pine DJ. Phase diagrams of nearly hard-sphere binary colloids. Phys Rev E 1995;52:4045-4057.

[104] (a) Imhof A, Dhont JKG. Experimental phase diagram of a binary colloidal hard-sphere mixture with a large size ratio. Phys Rev Lett 1995;75:1662-1665; (b) Imhof A, Dhont JKG. Long-time self-diffusion in binary colloidal hard-sphere dispersions. Phys Rev E 1995;52:6344-6357.

[105] Dijkstra M, van Roij R, Evans R. Direct simulation of the

-. phase behavior of binary hard-sphere mixtures: test of the depletion potential description. Phys Rev Lett 1999; 82:117-120.

[106] Dijkstra M, van Roij R, Evans R. Phase behavior and

-• $\quad$ structure of binary hard-sphere mixtures. Phys Rev Lett 1998;81:2268-2271.

[108] Dickman R, Attard P, Simonian V. Entropic forces in binary

-• hard sphere mixtures: theory and simulation. J Chem Phys 1997;107:205-213.

[109] Götzelmann B, Roth R, Dietrich S, Dijkstra M, Evans R.

•- Depletion potential in hard-sphere fluids. Europhys Lett 1999;47:398-404.

[110] Biben T, Bladon P, Frenkel D. Depletion effects in binary

-• hard-sphere fluids. J Phys: Condens Matter 1996;8: 10799-10821.

[111] Tehver R, Maritan A, Koplik J, Banavar JR. Depletion forces in hard-sphere colloids. Phys Rev E 1999;59: R1339-R1342.

[112] Mao Y, Cates ME, Lekkerkerker HNW. Depletion force in

- colloidal systems. Physica A 1995;222:10-24.

[113] Mendez-Alcaraz JM, Klein R. Depletion forces in colloidal mixtures'. Phys Rev. E 2000;61:4095-4099.

[114] Heno Y, Regnaut C. Hard-sphere colloidal suspension of macroparticles in a multicomponent solvent. J Chem Phys 1991;95:9204-9208.

[115] Amokrane. S. Influence of attractive forces on the solvent mediated potential of mean force between colloidal particles. J Chem Phys 1998;108:7459-7468.

[116] Götzelmann B, Evans R, Dietrich S. Depletion forces in

• fluids. Phys Rev E 1998;57:6785-6800.

[117] Roth R, Götzelmann B, Dietrich S. Depletion forces near curved surfaces. Phys Rev Lett 1999;83:448-451.

[118] Roth R, Evans R, Dietrich S. Depletion potential in hard-

- sphere mixtures: theory and applications. Phys Rev E 2000;62:5360-5377.

[119] Kaplan PD, Faucheux LP, Libchaber AJ. Direct observation of the entropic potential in a binary suspension. Phys Rev Lett 1994;73:2793-2796.

[120] (a) Dinsmore AD, Wong DT, Nelson P, Yodh AG. Hard spheres in vesicles: curvature-induced forces and particle-induced curvature. Phys Rev Lett 1998;80:409-412; (b) Crocker JC, Matteo JA, Dinsmore AD, Yodh AG. Entropic attraction and repulsion in binary colloids probed with a line optical tweezer. Phys Rev Lett 1999;82:4352-4355.

[121] Almarza NG, Enciso E. Phase equilibria of asymmetric hard

-• $\quad$ sphere mixtures. Phys Rev E 1999;59:4426-4433.

[122] Velasco E, Navascues G, Mederos L. Phase behavior of binary hard-sphere mixtures from perturbation theory. Phys Rev E 1999;60:3158-3164.

[123] Clement-Cottuz J, Amokrane S, Regnaut C. Phase diagram of highly asymmetric binary mixtures: a study of the role of attractive forces from the effective one-component approach. Phys Rev E 2000;61:1692-1702.

[124] Lue L, Woodcock LV. Depletion effects and gelation in a binary hard-sphere fluid. Mol Phys 1999;96:1435-1443.

[125] Malherbe JG, Amokrane S. True mixture vs. effective one component fluid models of asymmetric binary hard sphere mixtures: a comparison by simulation. Mol Phys 2001; 99:355-361.

[126] Poon WCK. Phase separation, aggregation, and gelation in

-• colloid-polymer mixtures and related systems. Curr Opinion Colloid Interface Sci 1998;3:593-599.

[127] Shaw MR, Thirumalai D. Free polymer in a colloidal solution. Phys Rev A 1991;44:R4797-R4800.

[128] (a) Fuchs M, Schweizer KS. Structure and thermodynamics

- of colloid-polymer mixtures: A macromolecular approach. Europhys Lett 2000;51:621-627; (b) Chatterjee AP, Schweizer KS. Influence of solvent quality and thermal fluctuations on polymer-mediated depletion interactions. Macromolecules 1999;32:923-934; (c) Chatterjee AP, Schweizer KS. Microscopic theory of polymer-mediated interactions between spherical particles. J Chem Phys 1998;109:10464-10476.

[129] Schweizer KS, Curro JG. Integral equation theories of the structure, thermodynamics, and phase transitions of polymer fluids. Adv Chem Phys 1997;98:1-142.

[130] (a) Meijer EJ, Frenkel D. Computer-simulation of Colloid

- $\quad$ polymer mixtures. Physica A 1995;213:130-137; (b) Meijer EJ, Frenkel D. Colloids dispersed in polymer-solutions-a computer-simulation study. J Chem Phys 1994;100: 6873-6887; (c) Meijer EJ, Frenkel D. Computer-simulation of polymer-induced clustering of colloids. Phys Rev Lett 1991;67:1110-1113.

[131] Ilett SM, Orrock A, Poon WCK, Pusey PN. Phys Rev E 1995;51:1344.

[133] Lekkerkerker HNW, Poon WCK, Pusey PN, Stroobants A,

- Warren PB. Phase behaviour of Colloid + polymer mixtures. Europhys Lett 1992;20:559-564.

[134] (a) Schmidt M, Löwen H, Brader JM, Evans R. Density

-• functional for a model colloid-polymer mixture. Phys Rev Lett 2000;85:1934-1937; (b) Brader JM, Evans R, Schmidt 
M, Löwen H. Phys Rev Lett (submission); (c) Evans R, Brader JM, Roth R, Dijkstra M, Schmidt M, Löwen H. Interfacial properties of model colloid-polymer mixtures. Phil Trans Roy Soc Ser A; (in press).

[135] Dijkstra M, Brader JM, Evans R. Phase behaviour and -• structure of model colloid-polymer mixtures. J Phys: Condens Matter 1999;11:10079-10106.

[136] (a) Dickman R, Yethiraj A. Polymer-induced forces between

-• colloidal particles - a Monte-Carlo simulation. J Chem Phys 1994;100:4683-4690; (b) Yethiraj A, Hall CK, Dickman $\mathrm{R}$. Interaction between colloids in solutions containing dissolved polymer. J Colloid Interf Sci 1992;151:102-117.

[137] Tuinier R, Vliegenthart GA, Lekkerkerker HNW. Depletion

- interaction between spheres immersed in a solution of ideal polymer chains. J Chem Phys 2000;113:10768-10775.

[138] Louis AA, Bolhuis PG, Hansen JP, Meijer. EJ. Can polymer

- coils be modelled as soft colloids? Phys Rev Lett 2000;85:2522-2525.

[139] Hanke A, Eisenriegler E, Dietrich S. Polymer depletion

- effects near mesoscopic particles. Phys Rev E 1999; 59:6853-6878.

[140] Roth R, Evans R. The depletion potential in non-additive

- $\quad$ hard-sphere mixtures. Europhys Lett 2001;53:271-277.

[141] (a) Ye X, Narayanan T, Tong P, Huang JS. Neutron Scattering study of depletion interactions in a colloid-polymer mixture. Phys Rev Lett 1996;76:4640-4643; (b) Ye X, Narayanan T, Tong P et al. Depletion interactions in colloid-polymer mixtures. Phys Rev E 1996;54:6500-6510.

[142] Ohshima YN, Sakagami H, Okumuto K et al. Direct measurement of infinitesimal depletion force in a colloid-polymer mixture by laser radiation pressure. Phys Rev Lett 1997;78:3963-3966.

[143] (a) Rudhardt D, Bechinger C, Leiderer P. Direct measurement of depletion potentials in mixtures of colloids and non-ionic polymers. Phys Rev Lett 1998;81:1330-1333; (b) Bechinger C, Rudhardt D, Leiderer P, Roth R, Dietrich S.
Understanding depletion forces beyond entropy. Phys Rev Lett 1999;83:3960-3963; (c) Rudhardt D, Bechinger C, Leiderer. P. Repulsive depletion interactions in colloid-polymer mixtures. J Phys: Condens Matt 1999;11:10073-10078.

[144] (a) Verma R, Crocker JC, Lubensky TC, Yodh AG. Entropic colloidal interactions in concentrated DNA solutions. Phys Rev Lett 1998;81:4004-4007; (b) Verma R, Crocker JC, Lubensky TC, Yodh. AG. Attractions between hard colloidal spheres in semiflexible polymer solutions. Macromolecules 2000;33:177-186.

[145] Gast AP, Hall CK, Russel WB. Polymer-induced phase

- $\quad$ separations in non-aqueous colloidal suspensions. J Colloid Interface Sci 1983;96:251-267.

[146] Brader JM, Dijkstra M, Evans R. Inhomogeneous model

-• colloid-polymer mixtures: adsorption at a hard wall. Phys Rev E; (in press).

[147] Brader JM, Evans R. The fluid-fluid interface of a model colloid-polymer mixture. Europhys Lett 2000;49:678-684.

[148] Soga KG, Melrose JR, Ball RC. Continuum percolation and depletion flocculation. J Chem Phys 1998;108:6026-6032.

[149] Dijkstra M. To be published.

• 150$]$ Moussaïd A, Poon WCK, Pusey PN, Soliva MF. Structure of marginal and fully developed colloidal liquids. Phys Rev Lett 1999;82:225-228.

\section{Further reading}

[107] (a) Attard P. Spherically inhomogeneous fluids. II. Hardsphere solute in a hard-sphere solvent. J Chem Phys 1989;91:3083-3089; (b) Attard P, Patey GN. Hypernettedchain closure with bridge diagrams - asymmetric hardsphere mixture. J Chem Phys 1990;92:4970-4982.

[132] Louis AA, Finken R, Hansen JP. The structure of - colloid-polymer mixtures. Europhys Lett 1999;46:741-747. 